\title{
Palliative treatment of uncontrollable hypercalcemia due to parathyrotoxicosis: denosumab as rescue therapy
}

\author{
Reiner Jumpertz von Schwartzenberg', Ulf Elbelt ${ }^{1}$, Manfred Ventz' ${ }^{1}$, Knut Mai', $^{1}$ \\ Tina Kienitz' ${ }^{1}$, Lukas Maurer', Thomas Rose ${ }^{2}$, Jens C Rückert ${ }^{3}$, Christian J Strasburger ${ }^{1}$ \\ and Joachim Spranger ${ }^{1}$
}

${ }^{1}$ Department of Endocrinology and Metabolic Diseases ${ }^{2}$ Division of Rheumatology and Clinical Immunology, Medical Department ${ }^{3}$ Department of General, Visceral, Vascular and Thoracic Surgery,

Charité - Universitätsmedizin, Charitéplatz 1, 10117 Berlin, Germany

\author{
Correspondence \\ should be addressed \\ to $\mathrm{R}$ Jumpertz von \\ Schwartzenberg \\ Email \\ reiner.jumpertz@charite.de
}

\begin{abstract}
Parathyroid carcinoma is a rare disease leading to severe hypercalcemia due to hyperparathyroidism. Surgery is the primary treatment option. A more progressive form of the disease is characterized by parathyrotoxicosis, and subsequent hypercalcemia is the most common cause of death. We report a case presenting with severe hypercalcemia due to parathyrotoxicosis from parathyroid carcinoma treated for the first time using the monoclonal antibody denosumab as a rescue therapy and present long-term follow-up data. The 71-year-old patient presented with severe hypercalcemia due to metastatic parathyroid carcinoma. Despite undergoing treatment with bisphosphonates, cinacalcet hydrochloride, and forced diuresis, the patient's condition deteriorated rapidly due to resistant hypercalcemia. Surgery performed because of spinal metastasis and forced diuresis lowered calcium levels, albeit they remained in the hypercalcemic range and significantly increased when forced diuresis was stopped. Considering a palliative situation to overcome hypercalcemia, we decided to administer denosumab, a monoclonal antibody that binds to the receptor activator of nuclear factor-kappa B ligand. After a single subcutaneous administration of $60 \mathrm{mg}$ denosumab, calcium levels normalized within one day. Subsequent denosumab injections led to permanent control of serum calcium for more than 2 years despite rising parathyroid hormone levels and repeated surgeries. Together with recent cases in the literature supporting our observation, we believe that denosumab is relevant for future trials and represents an effective tool to control hypercalcemia in patients with advanced stages of parathyroid cancer.
\end{abstract}

\section{Learning points:}

- Severe hypercalcemia is the most common cause of death in patients with parathyroid carcinoma.

- The monoclonal antibody denosumab rapidly lowered severely elevated serum calcium levels due to parathyrotoxicosis.

- Denosumab was effective in the long-term treatment of hypercalcemia despite progression of parathyroid carcinoma. 


\section{Background}

Parathyroid carcinoma is a rare condition occurring in $0.7-2.1 \%$ of patients with primary hyperparathyroidism. As of today, surgery is the only known curative treatment. However, pre-surgical differentiation of excess parathyroid hormone (PTH) due to parathyroid adenoma or carcinoma is challenging. Observational studies have demonstrated that $76.6-80 \%$ of patients with parathyroid carcinoma have a tumor size of $>3 \mathrm{~cm}$ and $85 \%$ had presurgical calcium concentrations of $>3 \mathrm{mmol} / \mathrm{l}$ (1). Thus, large tumors and severe hypercalcemia indicate higher risk for parathyroid cancer. If parathyroid cancer exists, en bloc resection of the tumor with centrocervical lymphadenectomy is the recommended treatment. Nonetheless, cancer is not suspected pre-surgically very often, and local excision is still the most common method of surgery despite higher risk rates of death and recurrence of the tumor $(1,2)$. In case of tumor recurrence, local resection of the tumor and metastases is the recommended treatment approach. In some cases reduction of tumor mass may also include radiotherapy, cryoablation, or embolization. In advanced stages of the disease, reduction of tumor mass may no longer be a feasible treatment option, and in these palliative cases, severe hypercalcemia represents the most common cause of death (3). Malignant PTH-driven hypercalcemia is the main cause of bone pain and pathological fractures in these patients, as well as pancreatitis, peptic ulcer, and anemia, and eventually leads to severe end-organ damage, such as renal faliure (4). The mainstay of palliative care for patients with advanced disease therefore includes control of bone loss and prevention of severe hypercalcemia to warrant quality of life. Various calcium-lowering drugs have been used in patients with parathyroid cancer such as mithramycin, plicamycin, gallium nitrate, and calcitonin combined with glucocorticoids leading to rather transient changes in calcium levels $(4,5)$. However, new drugs such as calcimimetics are more effective in lowering calcium levels and bear less side effects. Palliative medical therapy in parathyroid cancer patients today is mainly based on the use of intravenous bisphosphonates and calcimimetics such as cinacalcet hydrochloride.

Here, we report a case with severe parathyrotoxicosis due to advanced non-resectable parathyroid carcinoma treated with the additional administration of a new drug, the monoclonal antibody denosumab, for management of calcium levels

\section{Case presentation and treatment}

The 71-year-old male patient was first diagnosed with parathyroid cancer in 1992. Subsequent surgical treatment included local resection of the tumor and complete thyroidectomy leaving the patient with permanent palsy of the left laryngeal nerve. Histological analysis confirmed parathyroid carcinoma. Hereafter, the tumor was in remission for 13 years. In 2005, local recurrence of the tumor was observed in the thyroid bed. This was followed by complete resection and subsequent local radiation therapy. In December 2008, the patient underwent cervical lymphadenectomy dorsal to the right clavicle where no tumor tissue was found. One month later, the cancer metastasized cranially to the jugular notch of the sternum and was resected in toto. In 2009, osseous metastases occurred in the second rib on the left and seventh rib on the right side as well as in the body of the sternum. By the end of 2009, pulmonary masses highly suggestive of metastases were seen. Additionally, pulmonary embolism was diagnosed requiring subsequent anticoagulation. In 2010, the osseous metastases in the ribs and sternum were resected completely during three different interventions, followed by local radiation therapy. In 2011 pulmonary metastases remained stable, but after subsequent local progression had to be resected in toto by the beginning of 2012. For prevention of hypercalcemia and bone loss, the patient was treated with $4 \mathrm{mg}$ zolendronic acid intravenously every four weeks and $90 \mathrm{mg}$ daily oral administration of the calcimimetic cinacalcet hydrochloride. The patient did not tolerate higher doses of cinacalcet due to increased nausea. Nonetheless, in the course of the year 2012, a profound increase in serum calcium levels occurred accompanied by a rise in PTH levels (Fig. 1). Simultaneously, the patient suffered from increasing abdominal pain, nausea, and lost $\sim 10 \mathrm{~kg}$ of body weight over 1 year presenting a cachectic condition (54 kg body weight). By February 2013, serum calcium levels had risen to $3.7 \mathrm{mmol} / \mathrm{l}$ and PTH concentrations increased to $429 \mathrm{ng} / \mathrm{l}$ while the patient continued to suffer from severe abdominal pain, further weight loss, and obstipation. At this time, the patient was admitted to our inpatient unit for severe parathyrotoxicosis. During the course of the inpatient stay, forced diuresis with $3000 \mathrm{ml}$ of fluids ( $\mathrm{NaCl} 0.9 \%$ ) and $40 \mathrm{mg}$ of furosemide twice daily was administered for kidney protection and mainly for control of calcium levels. During this regimen, serum calcium levels decreased slightly; however, they remained at $3.0 \mathrm{mmol} / \mathrm{l}$ and thus above normal range (see Fig. 1). Nonetheless, the condition of the patient did not improve 
Serum calcium and parathyroid hormone over time

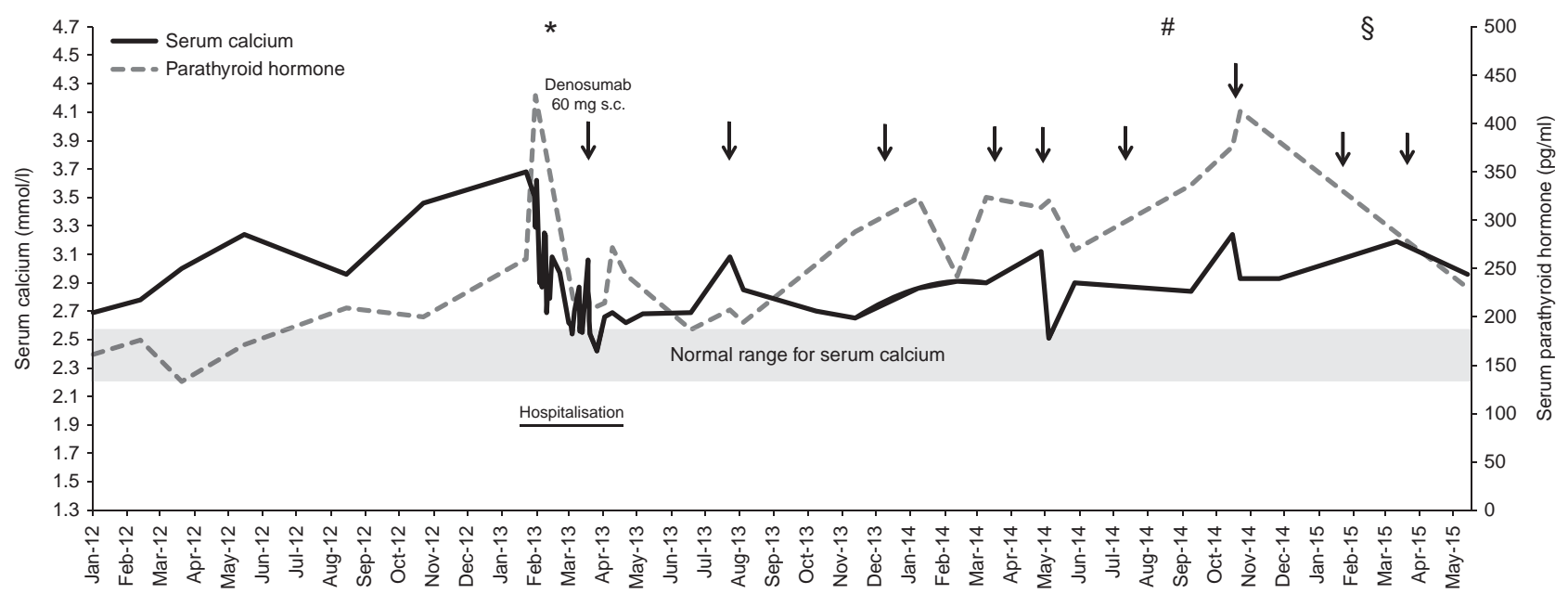

Figure 1

Serum calcium and parathyroid hormone levels over time. Albumincorrected serum calcium concentrations in $\mathrm{mmol} / \mathrm{l}$ and parathyroid hormone concentrations in our patient are depicted over time. Normal range for calcium is shown according to our laboratory standards (normal range for calcium: $2.20-2.55 \mathrm{mmol} / \mathrm{l}$; normal range for PTH: 10-65 ng/ml). Denosumab injections are indicated by black arrows; the patient additionally received $90 \mathrm{mg}$ of cinacalcet daily. In April 2014, cinacalcet

sufficiently due to additional onset of back pain. A CTcontrolled 18F-fluordesoxyglucose positron emission tomography (PET) showed increased glucose uptake in the sixth thoracic vertebra (Fig. 2). The patient underwent vertebral surgery for resection of the vertebral mass and dorsal stabilization. Histology confirmed a metastasis from parathyroid carcinoma. After surgery, PTH levels decreased to $210 \mathrm{ng} / \mathrm{l}$. However, despite forced diuresis and additional infusion of $4 \mathrm{mg}$ zolendronic acid, calcium levels declined only slightly and mostly remained in the hypercalcemic range. Review of the neck region in PETimaging raised suspicion of local recurrence on the right side of the thyroid bed, although no distinct activity peak could be identified (Fig. 3). However, venous catheterization confirmed excess PTH on the right side of the neck. Due to repeated surgery and subsequent scar tissue development in this area as well as left laryngeal nerve palsy, additional neck surgery did not seem beneficial for the patient. One month after vertebral surgery, calcium levels increased again to $3.1 \mathrm{mmol} / \mathrm{l}$ (Fig. 1). Taken together, the patient was facing rising calcium levels despite surgery, ongoing forced diuresis, intravenous bisphosphonate therapy, and daily oral administration of cinacalcet hydrochloride. At this point, we did not have further treatment options and searched the literature for dosage was increased to $180 \mathrm{mg}$ daily, which was not tolerated by the patient and subsequently continued at $90 \mathrm{mg}$ daily. * represents resection of spinal metastasis at vertebra 6 . \# represents resection of a supraclavicular metastasis on the left side without significant reduction in PTH levels. $\S$ represents resection of three cervical metastases on the left side. Forced diuresis comprised infusion of $2000-3000 \mathrm{ml}$ of $0.9 \%$ sodium chloride solution and $80 \mathrm{mg}$ furosemide daily.

alternative approaches. Based on an article by Boikos and Hammers who had recently used denosumab in a patient with hypercalcemia from metastatic renal cell carcinoma (6), we initiated the off-label administration of denosumab, a monoclonal antibody that binds to the receptor activator of nuclear factor-kappa B ligand (RANKL) in our patient. Denosumab is a bone mineral density modifying full human antibody $\left(\mathrm{IgG}_{2}\right)$ with high affinity to RANKL, thereby inhibiting the RANKL-RANK interaction. RANKL is secreted by osteoblasts upon stimulation by PTH leading to differentiation and stimulation of osteoclasts. Thus, denosumab inhibits RANKL-mediated bone resorption and thereby promotes reduction in serum calcium concentrations. We administered $60 \mathrm{mg}$ of denosumab subcutaneously in our patient.

\section{Outcome and follow-up}

Within one day after the denosumab injection, serum calcium levels normalized and remained stable despite discontinuation of forced diuresis (Fig. 1). We discharged the patient from our inpatient unit two days later. During regular outpatient visits, calcium levels remained stable between 2.6 and $2.7 \mathrm{mmol} / \mathrm{l}$ for four months (Fig. 1). During this time, the condition of the patient 
Palliative treatment of
ID: 15-0082; November 2015 DOI: $10.1530 / E D M-15-0082$

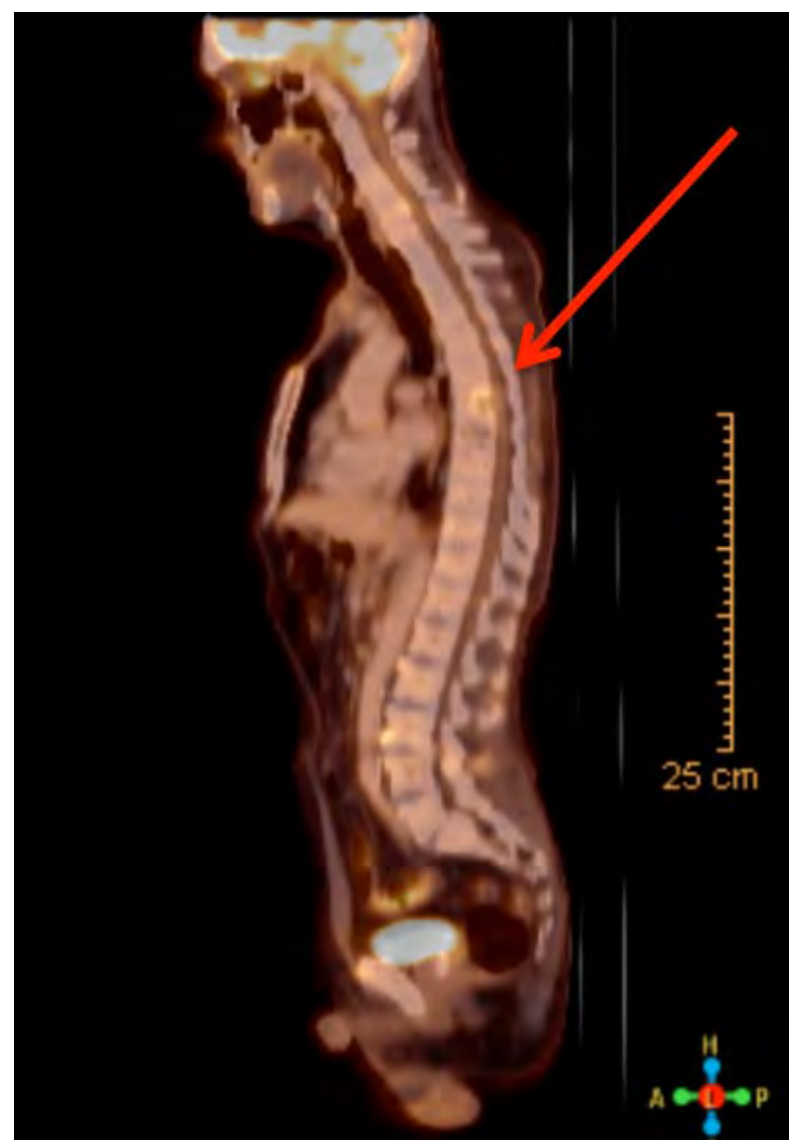

Figure 2

Sagittal PET-CT image of a spine metastasis. Location of spinal metastasis (red arrow) in a CT-merged 18F-fluordesoxyglucose positron emission tomography (PET).

considerably improved with reduced nausea and back pain and improved appetite and weight gain. After a new rise in calcium levels, another $60 \mathrm{mg}$ of denosumab were injected, yet again followed by reduced and stable serum calcium levels over months despite high PTH levels. In total, six doses of denosumab during the following months led to stable calcium levels without further need for inpatient treatment. By November 2014, PTH levels peaked at $413 \mathrm{pg} / \mathrm{ml}$; however, another denosumab injection sufficiently stabilized serum calcium. Clinically relevant side effects were not observed. Resection of a new supraclavicular metastasis on the left side in August 2014 did not affect PTH levels significantly. In February 2015, another three cervical metastases were removed. Again, after repeated Denosumab injections, calcium levels remained above normal but stable until the last followup in May 2015. The follow-up period included 25 months in total after the first denosumab injection.

\section{Discussion}

We reported a case presenting with severe treatmentresistant hypercalcemia secondary to parathyrotoxicosis that responded to administration of the RANKL-antibody denosumab with rapid and persistent reduction in serum calcium levels. We observed no relevant side effects.

Subcutaneous administration of the antibody has been proven to be effective in increasing bone mineral density and inhibiting bone resorption in post-menopausal women, as well as in reducing calcium levels in patients with hypercalcemia due to hematologic cancers or bone metastases of solid tumors $(7,8)$. However, to date, no clinical studies have addressed the efficacy of denosumab in reducing calcium levels in patients with severe PTH excess due to parathyroid carcinoma. One interesting aspect of this case is how rapidly calcium levels dropped to normal ranges despite ongoing parathyrotoxicosis. However, during this phase, we cannot clearly discriminate the calcium lowering effects of the surgery, zolendronic acid, and denosumab. Calcium levels had been increasing despite zolendronic acid infusions over the previous months, and although calcium levels decreased after surgery, they remained in the hypercalcemic range and could not be further reduced by forced diuresis. The temporal association of denosumab injection and drop in calcium levels is highly indicative of an acute effect of denosumab. An early case report by Boikos and Hammers from 2012 demonstrated calcium lowering effects of denosumab in a patient with pulmonary metastases of

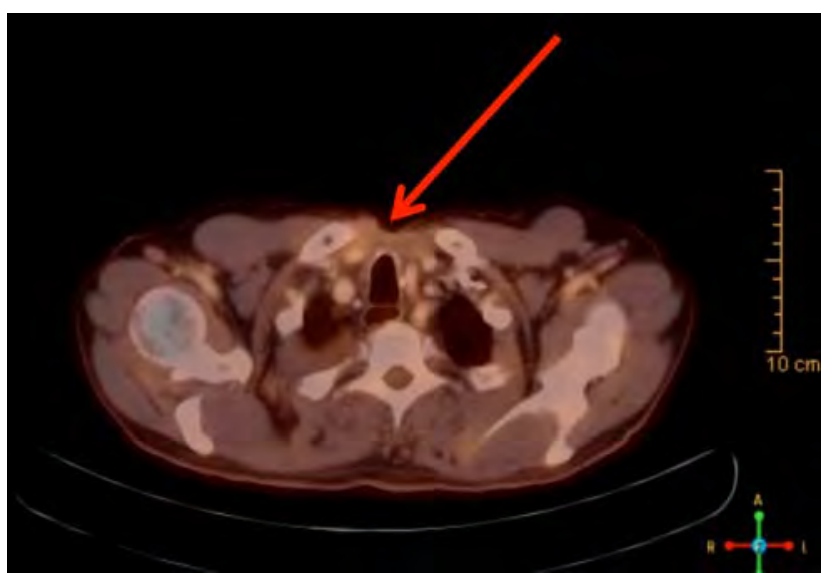

Figure 3

Transverse PET-CT image of the neck/thorax chest region. Transverse picture of an area in the neck/chest region with potentially increased local glucose uptake (red arrow) of a CT-merged 18F-fluordesoxyglucose positron emission tomography (PET), suspicious but not conclusive for local recurrence. 
Table 1 Current literature on the use of denosumab in hypercalcemia due to parathyrotoxicosis

\begin{tabular}{l} 
References \\
\hline$(6,8)$ \\
$(9)$ \\
$(11)$ \\
$(12)$ \\
$(10)$ \\
Present Case \\
$\quad$ Report
\end{tabular}

\begin{tabular}{l} 
Hypercalcemia due to \\
\hline Parathyroid hormone \\
related peptide \\
Parathyroid hormone \\
Parathyroid hormone \\
Parathyroid hormone \\
Parathyroid hormone \\
Parathyroid hormone
\end{tabular}

\begin{tabular}{l} 
Origin \\
\hline Renal cell carcinoma \\
Parathyroid carcinoma \\
Parathyroid carcinoma \\
Parathyroid carcinoma \\
Parathyroid carcinoma \\
Parathyroid carcinoma
\end{tabular}

\begin{tabular}{c} 
Injections \\
\hline 1 \\
3 \\
15 \\
7 \\
5 \\
9 \\
\hline
\end{tabular}

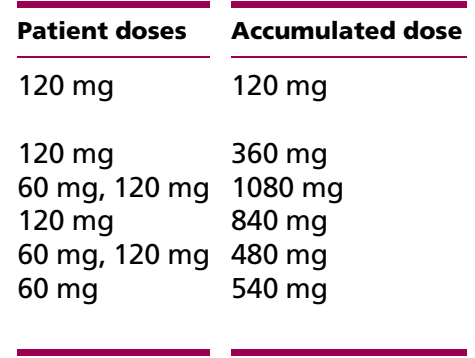

Follow-up

1 month

10 months

23 months

14 months

7 months

26 months

Comparison of the current literature regarding denosumab application, dosage, and follow-up time.

renal cell carcinoma, who suffered from severe hypercalcemia secondary to excess of PTH-related peptide. Interestingly, similar to our observation, calcium levels dropped rapidly within 2 days after denosumab administration and remained stable for about a month (6). A similar observation was made in a patient with parathyroid cancer, where the authors used much higher initial doses of denosumab. Again, calcium levels dropped rapidly despite rising PTH levels (9). Unfortunately, longer follow-up was not possible because the patient died due to disease progression. Recently, Karruppiah et al. have made similar observations in a patient with parathyrotoxicosis due to non-localizable parathyroid carcinoma using higher doses $(120 \mathrm{mg})$ of denosumab (10). Another important learning point however is that repeated denosumab injections in relatively low doses, such as in our case, can be sufficient for maintaining controlled calcium levels over months or even years (although surgical interventions most likely had an additional impact). This is supported by a recent case report, where the authors showed rapid declines in serum calcium upon denosumab administration and persistent normocalcemia over months (11). After recurring calcium peaks due to rising PTH levels, higher doses of denosumab and more frequent applications again lead to normalization of serum calcium. Additionally, Itoshima et al. have also used denosumab in a patient with even more severe parathyrotoxicosis than in our case, and frequent administration of denosumab, albeit in a higher dosage $(120 \mathrm{mg})$, was sufficient to maintain normal calcium levels (12). In our case, denosumab dosage was lower and administration frequency was marginally higher; however, calcium levels were slightly above normal, and thus, higher doses also seem feasible in our patient. Together with Vellanki et al., our case is the only one with a long term follow-up after the first denosumab injection. For an overview of the current literature and denosumab dosages see Table 1 .
Taken together, 1) subcutaneous application of denosumab led to rapid and permanent reduction in serum calcium levels in our case with parathyrotoxicosis secondary to parathyroid carcinoma. 2) Long term followup showed that repeated low-dose denosumab injections were effective in maintaining stable calcium levels despite excess PTH levels from parathyroid carcinoma.

Declaration of interest

The authors declare that there is no conflict of interest that could be perceived as prejudicing the impartiality of the research reported.

\section{Funding}

This research was funded by in-house resources of the Department of Endocrinology, Diabetes and Nutrition; Charité-Universitätsmedizin; and by the Charité Clinical Scientist Program.

\section{Patient consent}

Written informed consent was obtained from the patient for publication of this case report and the accompanying images.

\section{Author contribution statement}

$\mathrm{R}$ Jumpertz von Schwartzenberg: treatment of the patient, decisionmaking for denosumab use, follow-up, preparation of the manuscript; $\mathrm{U}$ Elbelt: treatment of the patient, decision-making for denosumab use, follow-up, critical review of the manuscript; $M$ Ventz: follow-up, critical review of the manuscript; K Mai: treatment of the patient, critical review of the manuscript; $T$ Kienitz and L Maurer: treatment of the patient, critical review of the manuscript; T Rose: decision-making for denosumab use, critical review of the manuscript; C J Strasburger and J Spranger: treatment of the patient, decision making for denosumab use, critical review of the manuscript.

\section{Acknowledgements}

We kindly appreciate the agreement of the patient for publication of these data. Additionally, we thank the nursing staff that formerly worked in ward 142 of the Charité University Hospital in Berlin Campus Mitte. 
Endocrinology

Diabetes \& Metabolism

CASE REPORTS
R Jumpertz von

Schwartzenberg and others
Palliative treatment of

uncontrollable hypercalcemia
ID: 15-0082; November 2015 DOI: 10.1530/EDM-15-0082

\section{References}

1 Talat N \& Schulte KM 2010 Clinical presentation, staging and longterm evolution of parathyroid cancer. Annals of Surgical Oncology 17 2156-2174. (doi:10.1245/s10434-010-1003-6)

2 Schulte KM, Talat N, Miell J, Moniz C, Sinha P \& Diaz-Cano S 2010 Lymph node involvement and surgical approach in parathyroid cancer. World Journal of Surgery 34 2611-2620. (doi:10.1007/ s00268-010-0722-y)

3 Wei CH \& Harari A 2012 Parathyroid carcinoma: update and guidelines for management. Current Treatment Options in Oncology 13 11-23. (doi:10.1007/s11864-011-0171-3)

4 Shane E 2001 Clinical review 122: parathyroid carcinoma. Journal of Clinical Endocrinology and Metabolism 86 485-493. (doi:10.1210/jcem. 86.2.7207)

5 Harari A, Waring A, Fernandez-Ranvier G, Hwang J, Suh I, Mitmaker E, Shen W, Gosnell J, Duh QY \& Clark O 2011 Parathyroid carcinoma: a 43-year outcome and survival analysis. Journal of Clinical Endocrinology and Metabolism 96 3679-3686. (doi:10.1210/jc.2011-1571)

6 Boikos SA \& Hammers HJ 2012 Denosumab for the treatment of bisphosphonate-refractory hypercalcemia. Journal of Clinical Oncology 30 e299. (doi:10.1200/JCO.2012.41.7923)

7 Hu MI, Glezerman I, Leboulleux S, Insogna K, Gucalp R, Misiorowski W, Yu B, Ying W \& Jain RK 2013 Denosumab for patients with persistent or relapsed hypercalcemia of malignancy despite recent bisphosphonate treatment. Journal of the National Cancer Institute 105 1417-1420. (doi:10.1093/jnci/djt225)

8 McClung MR, Lewiecki EM, Cohen SB, Bolognese MA, Woodson GC, Moffett AH, Peacock M, Miller PD, Lederman SN, Chesnut CH et al. 2006 Denosumab in postmenopausal women with low bone mineral density. New England Journal of Medicine 354 821-831. (doi:10.1056/ NEJMoa044459)

9 Bowyer SE, White AM, Ransom DT \& Davidson JA 2013 Resistant hypercalcaemia in metastatic parathyroid carcinoma. Medical Journal of Australia 198 559-561. (doi:10.5694/mja12.11243)

10 Karuppiah D, Thanabalasingham G, Shine B, Wang LM, Sadler GP, Karavitaki N \& Grossman AB 2014 Refractory hypercalcaemia secondary to parathyroid carcinoma: response to high-dose denosumab. European Journal of Endocrinology 171 K1-K5. (doi:10.1530/ EJE-14-0166)

11 Vellanki P, Lange K, Elaraj D, Kopp PA \& El Muayed M 2014 Denosumab for management of parathyroid carcinoma-mediated hypercalcemia. Journal of Clinical Endocrinology and Metabolism 99 387-390. (doi:10.1210/jc.2013-3031)

12 Itoshima S, Yuno A, Kato T, Kamada H, Ikota A, Usui A, Shimatsu A \& Koizumi S 2015 Denosumab for the treatment of refractory hypercalcemia in metastatic parathyroid carcinoma. AACE Clinical Case Reports 1 e141. (doi:10.4158/EP14449.CR)

Received in final form 23 October 2015

Accepted 29 October 2015 